\title{
Cerebral fat embolism after bilateral total knee replacement arthroplasty -A case report-
}

\author{
Ri-Na Chang, Jong-Hak Kim, Heeseung Lee, Hee-Jung Baik, Rack Kyung Chung, Chi Hyo Kim, and \\ Tae-Hu Hwang \\ Department of Anesthesiology and Pain Medicine, College of Medicine, Ewha Womans University, Seoul, Korea
}

Fat embolism syndrome is a rare and potentially lethal complication most commonly seen in long bone fractures and intramedullary manipulation. The clinical triad of fat embolism syndrome consists of mental confusion, respiratory distress, and petechiae. This study reports a case of cerebral fat embolism syndrome following elective bilateral total knee replacement. After an uneventful anesthesia and initial recovery, the patient developed neurologic symptoms nine hours postoperatively. (Korean J Anesthesiol 2010; 59: S207-S210)

Key Words: Arthroplasty, Fat embolism, Total knee replacement.

Fat embolism syndrome is a change in physiology resulting from mechanical causes, trauma, or sepsis. Fat globules generated within the systemic circulation induce pulmonary dysfunction, neurological changes, dermal symptoms, and dysfunction of several other organs. Although very rare, fat embolism syndrome is a fatal disease that develops in 1272 hours. Acute dyspnea may appear, and if not treated appropriately, may progress to cardiac arrest in rare cases $[1,2]$. However, due to symptom diversity and unclear diagnostic methods, it is difficult to treat the fat embolism correctly. This paper reports on the case study of a patient who developed sudden dyspnea and deterioration of the nervous system 9 hours after bilateral total knee replacement arthroplasty. The subsequent diagnosis of brain fat embolism syndrome was determined, appropriate treatments were administered, and the patient recovered without sequelae. This study recounts this case, particularly for anesthesiologists, to emphasize the necessity of comprehensive patient management even after surgery for risk group patients.

\section{Case Report}

A 74-year-old female patient (height: $157 \mathrm{~cm}$, weight: $56.8 \mathrm{~kg}$ ) was diagnosed with degenerative osteoarthritis and admitted for bilateral total knee replacement arthroplasty. The patient was also previously diagnosed with angina and hypertension 2 years earlier and placed on drug treatment (isosorbide-5-mininitrate and atenolol). Blood and biochemistry tests performed prior to surgery were within normal ranges. Similarly, on simple chest radiograph, no special findings were observed except

Received: May 24, 2010. Revised: 1st, June 26, 2010; 2nd, July 1, 2010. Accepted: July 21, 2010.

Corresponding author: Jong-Hak Kim, M.D., Department of Anesthesiology and Pain Medicine, College of Medicine, Ewha Womans University, 911-1, Mok-dong, Yangcheon-gu, Seoul 158-710, Korea. Tel: 82-2-2650-5285, Fax: 82-2-2655-2924, E-mail: kjhanes@ewha.ac.kr

(c) This is an open-access article distributed under the terms of the Creative Commons Attribution Non-Commercial License (http:// creativecommons.org/licenses/by-nc/3.0/), which permits unrestricted non-commercial use, distribution, and reproduction in any medium, provided the original work is properly cited. 
cardiomegaly (cardiothoracic ratio $=0.57$ ). Sinus bradycardia, first degree atrioventricular block, and T wave inversion in leads V2-3 were detected on electrocardiogram, and the ejection fraction by cardiac ultrasonography indicated a normal reading of $60 \%$.

To perform surgery under combined spinal epidural (CSE) anesthesia, $9 \mathrm{mg}$ 0.5\% high density bupivacaine (Marcaine ${ }^{\circledR}$, Astrazeneca, Sweden) was injected into the subarachnoid space, and an epidural catheter was inserted through which a test dose of $3 \mathrm{ml} 0.375 \%$ levobupivacaine (Chirocaine ${ }^{\circledR}$, Abbott, Norway) containing epinephrine diluted to $1: 200,000$ was injected. Surgery began after confirming that sensation was blocked to the 10th thoracic segment. During surgery, $5 \mathrm{~L} / \mathrm{min}$ $100 \%$ oxygen was administered via face mask. Vital signs of patient were stable throughout the entire surgery. Pulse oxygen saturation was maintained at $100 \%$, and arterial blood gas analysis performed every 50 minutes was also within normal ranges. Operation time was 3 hours and 5 minutes, and the total anesthesia period was 4 hours and 25 minutes. Estimated blood loss was approximately $1,100 \mathrm{ml}$, and urine volume was approximately $850 \mathrm{ml}$. During surgery, $500 \mathrm{ml}$ 6\% hydroxyethyl starch (Hextend $^{\circledR}$, Bio time, USA), 3,000 ml Hartmann solution, and 2 units of packed red blood cells (RBC) were administered. After surgery was completed, the patient was transported to a recovery room, and $6 \mathrm{~L} / \mathrm{min} 100 \%$ oxygen was supplied via face mask. After confirming that sensation was recovered up to the 11th thoracic nerve segment, pain was controlled by administering $5 \mathrm{ml} / \mathrm{h} 0.1 \%$ levobupivacaine and fentanyl (1.5 $\mu \mathrm{g} / \mathrm{ml}$ ) through the epidural catheter. In the recovery room, the patient showed stable vital signs, and arterial blood gas analysis was within normal ranges. The patient was transported to a ward with $100 \%$ oxygen supply of $2 \mathrm{~L} / \mathrm{min}$ via nasal cannula.

Immediately after transporting the patient to a ward, pulse oxygen saturation level was observed to be $90 \%$. Thus, $5 \mathrm{~L} / \mathrm{min}$ $100 \%$ oxygen was administered via face mask until pulse oxygen saturation level was again within normal ranges. Suddenly, nine hours after surgery, the patient exhibited a drowsy mental state during which she could not respond to calling. The patient was only able to open her eyes and respond to pain. Blood pressure and heart rate were within normal range, respiratory rate was 20 times/min, and pulse oxygen saturation level was $90 \%$. Thus, the rate of $100 \%$ oxygen supplied via face mask was raised to $7 \mathrm{~L} / \mathrm{min}$, and simple chest radiography and brain computed tomography (brain CT) were performed. Simple chest radiography revealed bilateral pulmonary effusion, but no abnormal findings were observed by brain CT. Afterward, patient symptoms were persistent, so, on the next day, brain magnetic resonance imaging (brain MRI) and 3D pulmonary arteriography were performed to rule out pulmonary embolism. Several bilateral small high signal intensity lesions in the cerebral and cerebellar cortices were detected on diffusion MRI. The apparent diffusion coefficient (ADC) in the area was reduced, suggesting fat embolism (Fig. 1). On 3D pulmonary arteriography, no special findings were detected.

Upon referral to the neurology department, the patient was transferred to the stroke unit the day after surgery. Immediately after transfer, her breathing became shallow and irregular and pulse oxygen saturation level decreased to $84 \%$. She also exhibited eyeball deviation to the left side and nystagmus. The patient was unresponsive to even strong stimulation, and the conscious state was stuporous. The patient was intubated because, while being supplied with $7 \mathrm{~L} / \mathrm{min} 100 \%$ oxygen via face mask, pulse oxygen saturation level decreased to $58 \%$ and blood pressure decreased to $70 / 40 \mathrm{mmHg}$. The patient was transferred to the intensive care unit, and placed on a mechanical ventilator. Blood pressure was measured at 95/60 and, at that time, a fever of $38^{\circ} \mathrm{C}$ was observed. After arriving on the intensive care unit, the neurology department considered administering low molecular weight heparin. Nonetheless, blood tests performed at that time revealed that hemoglobin was $8.3 \mathrm{~g} / \mathrm{dl}$ and platelets had decreased to $34,000 / \mu \mathrm{l}$. In a blood coagulation test, an increase in coagulation time was indicated; therefore the drug could not be administered. Packed RBCs and platelets were transfused, and inotropics as well as diuretics were administered. Three days after surgery, ischemic colitis was observed, and a petechial rash developed all over the entire body, including the abdomen.

Four days after surgery, blood coagulation test results were normal, so administration of low molecular weight heparin was initiated. However, the conscious state of patient was still stuporous, and neurological symptoms were unimproved. Five days after surgery, an electroencephalogram was performed from which moderate to severe diffuse cerebral dysfunction was found.

Seven days after surgery, due to improved conscious state
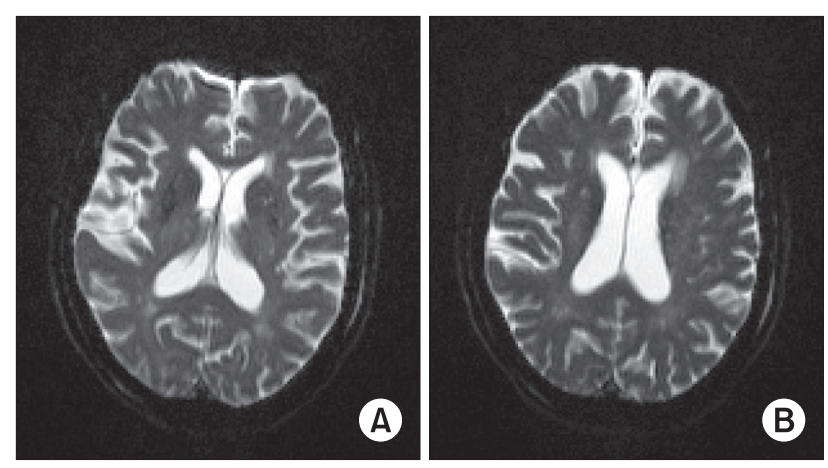

Fig. 1. Diffusion weighted images (A, B) reveal multifocal high signal intensity change in the bilateral cerebral hemisphere and cerebellum. 
and arterial blood gas analysis, the patient was weaned off the mechanical ventilator. Thirteen days after surgery, the endotracheal tube was removed, and respiratory and neurological symptoms were improved. Seventeen days after surgery, the patient was transferred to a general ward. Twenty six days after surgery, brain MRI revealed that multiple brain infarctions had become normal, and the patient was discharged 44 days after surgery.

\section{Discussion}

It has been shown that fat embolism syndrome is caused by fracture of the ilium or other pelvic bones, and multiple trauma. However, other possible causes include severe burns, infection, kidney transplant, liposuction, cardiopulmonary bypass, and transfusions. Moreover, although rare, cases of fat embolism syndrome after gastrectomy have been reported [3-7].

Based on clinical symptoms, fat embolism syndrome is diagnosed by eliminating other possible causes. Major clinical symptoms are respiratory distress symptoms, central nervous system symptoms, and dermal symptoms. Such symptoms may be induced by various causes other than fat embolism syndrome, and thus differentiation is required. Pulmonary embolism or pneumonia may cause dyspnea and hypoxemia while hypoxemia or meningitis may cause deterioration of brain function [1]. Since our patient had a past history of ischemic heart disease, myocardial infarction or cardiac shock were considered as causes. However, since surgery was performed under CSE anesthesia, and blood pressure, pulse, electrocardiogram, oxygen saturation level, and arterial blood gas analysis were within normal ranges throughout the entire anesthesia period, these causes could be ruled out. In $75 \%$ of fat embolism syndrome patients, respiratory distress symptoms are detected first, with $10 \%$ progressing to respiratory failure [8]. Similarly, in our case study, dyspnea and hypoxemia were

Table 1. Gurd's Criteria for Diagnosis of Fat Embolism Syndrome

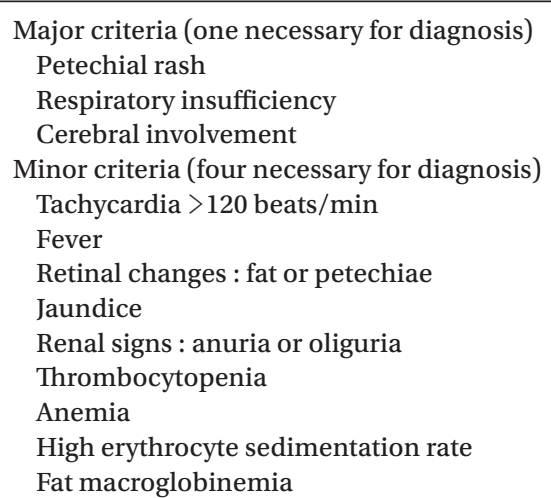

From Gurd and Wilson's study [5]. detected first. Regrettably, aggressive oxygenation treatments through immediate arterial blood gas analysis were not performed. Alteration of brain function is detected in $86 \%$ of patients, and the pattern varies from drowsy mental state to coma [7]. In $20-50 \%$ of patients, petechial rash is detected in the conjunctiva, around the neck, the axillary area, and the upper body. It appears within 24-36 hours and resolves spontaneously within one week [1].

Several investigators have reported criteria mainly based on clinical symptoms for the diagnosis of fat embolism syndrome. Criteria developed by Gurd and Wilson in 1974 [5] are widely used (Table 1). At least one of the major criteria and four of the minor criteria are necessary for diagnosing fat embolism syndrome [5]. Our patient exhibited all of the major symptoms of Gurd's diagnostic criteria, as well as the minor criteria of tachycardia, oliguria, fever of $38^{\circ} \mathrm{C}$, thrombocytopenia, and the elevation of erythrocyte sedimentation rate.

Radiological findings are also useful for diagnosis. Brain CT shows normal findings in most cases. As brain MRI is more sensitive, small high signal intensity lesions scattered in the cerebral white matter, cerebellum, and brain stem are detected on T2 weighted imagings or diffusion weighted imagings $[4,6,7]$. In our case, brain CT revealed no specific findings. Nevertheless, on T2 weighted imagings and diffusion weighted imaging of MRI, many bilateral small lesions in the cerebral white matter and the cerebellum were observed.

In fat embolism syndrome, prevention, early detection, and appropriate treatment are important. First, anesthesiologists and surgeons should recognize patients at-risk for developing fat embolism syndrome. Patients considered high risk for the development of fat embolism syndrome are both young and old, with closed or multiple fractures, as well as patients treated with only conservative therapies for iliac fracture. Particularly, it has been reported that in cases of bilateral total knee replacement arthroplasty with long tourniquet time, the risk of developing fat embolism syndrome increases [9]. In contrast, types of anesthesia, past medication history of patient prior to surgery, and type or duration of patient controlled analgesia are not associated with complications or development of cognitive dysfunction after knee arthroplasty [10]. When performing surgery on high risk patients, continuous hemodynamic monitoring by arterial cannulation and regular arterial blood gas analysis should be performed in addition to basic monitoring. In bilateral total knee replacement arthroplasty, measurement of pulmonary arterial blood pressure is also useful as a means of diagnosis and prognosis. In a recent study, during surgery, if cardiovascular system instability caused by fat embolism is detected, it is recommended to maintain perfusion pressure and use vasoconstrictors to maintain the function of the right sided heart [11]. After surgery, similarly, in conjunction 
with basic pulse oxygen saturation level monitoring, comprehensive monitoring of patients through regular arterial blood gas analysis should detect the early deterioration of the respiratory system of patient, and sufficient oxygen should be supplied. In our case, bilateral total knee replacement arthroplasty was performed, and a tourniquet was used for the right and left sides for 55 minutes each, which is thought to raise the risk of fat embolism syndrome. In addition, considering that consciousness altered suddenly nine hours after surgery, patients with high risk for the development of fat embolism syndrome, even without the presentation of special problems during surgery and anesthesia, should be sent to intensive care unit for continuous observation and early detection of symptoms as well as rapid and appropriate treatment.

It is important to perform conservative treatments for hypoxemia, hypotension, and reduction of the blood flow to peripheral organs simultaneously for the treatment of fat embolism syndrome. Among these treatments, securing the airway and oxygenation in tissues through oxygen supply are important [5]. In arterial blood gas analysis, $\mathrm{PaO}_{2}$ higher than $90 \mathrm{mmHg}$ should be maintained, and if required, endotracheal intubation and mechanical ventilation can be considered. Moreover, appropriate hydration is also vital. If effective blood volume within blood vessels is not maintained, pulmonary injury caused by fat embolism may be exacerbated. Albumin administration is helpful for maintaining the blood volume. In addition, it has been reported that early administration of highdose steroid reduces the development of hypoxemia and fat embolism. Nonetheless, therapeutic and prophylactic effects of high dose steroids are still controversial [12,13]. It was reported that heparin [14] and dextran [15] exerted fat degradation effects that help treatments, but it was found ineffective in subsequent studies. Hence, further studies are required [11].

In our patient, the department of neurology recommended the administration of low molecular weight heparin during the early onset period, but because of the risk of postsurgical hemorrhage and impediment of wound healing, treatments for brain fat embolism syndrome were limited. In addition, during surgery, regional anesthesia was performed and the epidural patient controlled analgesia method (PCA) was applied. Hence, potential hemorrhage to the epidural space should have been considered. We consider that, for brain fat embolism cases, anesthesiologists, neurologists, and surgeons should communicate closely, and perform the most appropriate treatments for patients.

Our case study was a patient who developed brain fat embolism after total knee replacement arthroplasty. If such patients show a sudden deterioration of consciousness, a fat embolism with a fatal prognosis should be suspected. As shown in our case, since symptoms may manifest even nine hours after surgery, anesthesiologists should recognize high risk patients by presurgical evaluation and aggressive efforts be made to obtain relevant information on patients in advance. To prevent fat embolism syndrome, risk factors should be corrected if possible, and careful anesthetic management should be undertaken. Furthermore, early diagnosis and appropriate management of fat embolism are important and prior to and following surgery, patients should be monitored comprehensively in the intensive care unit.

\section{References}

1. Shaikh N. Emergency management of fat embolism syndrome. J Emerg Trauma Shock 2009; 2: 29-33.

2. Fulde GW, Harrison P. Fat embolism--a review. Arch Emerg Med 1991; 8: 233-9.

3. Hwang KI, Sohn JT, Kang SJ, Kim HB, Lee HK, Chung YK. On fat embolism syndrome occurred in the recovery room after an intramedullary nailing of femur fracture: a case report. Korean J Anesthesiol 1999; 37: 731-6.

4. Ihn YK, Baik JH. Cerebral fat embolism as a rare complication of postgastrectomy: case report. J Korean Radiol Soc 2004; 51: 591-4.

5. Gurd AR, Wilson RI. The fat embolism syndrome. J Bone Joint Surg Br 1974; 56B: 408-16.

6. Shaikh N, Parchani A, Bhat V, Kattren MA. Fat embolism syndrome: clinical and imaging considerations: case report and review of literature. Indian J Crit Care Med 2008; 12: 32-6.

7. Jenkins K, Chung F, Wennberg R, Etchells EE, Davey R. Fat embolism syndrome and elective knee arthroplasty. Can J Anaesth 2002; 49: 19-24.

8. Johnson MJ, Lucas GL. Fat embolism syndrome. Orthopedics 1996; 19: 41-50.

9. Adili A, Bhandari M, Petruccelli D, De Beer J. Sequential bilateral total knee arthroplasty under 1 anesthetic in patients $>$ or $=75$ years old: complications and functional outcomes. J Arthroplasty 2001; 16: 271-8.

10. Sulek CA, Davies LK, Enneking FK, Gearen PA, Lobato EB. Cerebral microembolism diagnosed by transcranial Doppler during total knee arthroplasty: correlation with transesophageal echocardiography. Anesthesiology 1999; 91: 672-6.

11. Byrick RJ. Fat embolism and postoperative coagulopathy. Can J Anaesth 2001; 48: 618-21.

12. Findlay JM, DeMajo W. Cerebral fat embolism. Can Med Assoc J 1984; $131: 755-7$.

13. Bederman SS, Bhandari M, McKee MD, Schemitsch EH. Do corticosteroids reduce the risk of fat embolism syndrome in patients with long-bone fractures? A meta-analysis. Can J Surg 2009; 52: 386-93.

14. Sage RH, Tudor RW. Treatment of fat embolism with heparin. $\mathrm{Br}$ Med J 1958; 1: 1160-1.

15. Bergentz SE, Gelin LE, Rudenstam CM, Zederfeldt B. Indications for the use of low viscous dextran in surgery. Acta Chir Scand 1961; 122: 343-57. 